\title{
RETROSPECTIVE STUDIES IN SCLERODERMA: EFFECT OF POTASSIUM PARA-AMINOBENZOATE ON SURVIVAL
}

\author{
Chris J. D. Zarafonetis, Lyubica Dabich, Donald Negri, \\ Joyce J. SKovronski, EdWard B. DeVol and Robert Wolfe \\ Department of Internal Medicine, The University of Michigan Medical School, \\ and the Department of Biostatistics, The University of Michigan School of Public Health, \\ Ann Arbor, Michigan, U.S.A.
}

(Received in revised form 12 March 1987)

\begin{abstract}
Demographic and survival data are presented for 390 patients with scleroderma. For the entire group an estimated $81.4 \%$ survived 5 years from diagnosis and $69.4 \%$ survived 10 years. Life-table analyses revealed that adequate treatment with potassium para-aminobenzoate (Potaba KPAB) was associated with improved survival $(p<0.01) ; 88.5 \% 5$ year survival rate and $76.6 \%$ 10 year survival rate for adequately treated patients. Five and ten year survival rates for patients never treated with KPAB were 69.8 and $56.6 \%$, respectively. Similar findings were obtained by comparing observed to expected mortality for these patients; again, KPAB therapy showed prolongation of survival. The Cox proportional hazards model was also applied to this retrospective study adjusting for baseline clinical involvement, demographics and KPAB treatment. There were some interesting results including a high significance for skin involvement per se as a prognostic indicator: the greater the extent of skin involvement the poorer prognosis. Time from first diagnosis to first University Hospital visit or admission when included as a covariate did not influence survival.
\end{abstract}

Scleroderma Retrospective study Potassium para-aminobenzoate therapy Improved survival

\section{INTRODUCTION}

The purpose of this report is to present survival data for a group of 390 patients with scleroderma who had been seen at The University of Michigan Medical Center during the period 1948 to July 1980 . In the initial paper of this series [1] the patient population of this retrospective study was characterized, and the softening effect of therapy with potassium paraaminobenzoate (Potaba ${ }^{\circledR}, \quad$ KPAB) on sclerodermatous skin was confirmed. One of the main objectives of the analyses herein reported is to examine whether there were differences in survival consequent to treatment or nontreatment with potassium para-aminobenzoate. In addition, analyses were performed to determine whether survival was affected by duration of the disease at the time of entry into the study,

Requests for reprints should be addressed to: Chris J. D. Zarafonetis, M.D., 2721 Bedford Road, Ann Arbor, MI 48104, U.S.A. and for possible prognostic significance of specified clinical findings at time of entry. Results of these analyses form the basis of this report.

\section{MATERIALS AND METHODS}

The methods used to identify and characterize this population of 390 scleroderma patients were detailed in the initial report of this series [1]. Data were extracted according to protocol for each clinic visit or admission to the University of Michigan hospital. The protocol listed 479 variables for which entries could be coded. Follow-up information was augmented by extensive correspondence and telephone contact. These patients came from widely scattered areas with most from Michigan, Ohio, Indiana, Illinois and Pennsylvania. Patients were generally referred by outside physicians to the Department of Internal Medicine and/or its sub-specialty sections of Rheumatology or Hematology (Simpson Memorial Institute, to 
the Department of Dermatology, or to the Department of Pediatrics.

All patients satisfied at entry the diagnostic criteria for systemic scleroderma. Patients with scleroderma plus aspects of "mixed connective tissue disease", lupus erythematosus, dermatomyositis, etc. were excluded from this analysis, however, since these associated manifestations would complicate interpretation of subsequent organic changes that might also be attributable to scleroderma. The group of 390 patients with scleroderma alone made 3746 coded visits (including admissions) to the University Hospital, an average of 9.6 per patient. These patients were followed from diagnosis for a total of 3394.6 years, an average of 8.71 years per patient. There were 208 patients with recorded visits (either an actual visit or a record entry indicating the patient's death status had been ascertained through the follow-up procedure) after 31 December 1978 . Only 40 patients were lost to follow-up prior to that date. Among the latter, loss of four occurred during the period 1951-1956, another 20 were lost during 1962-1972, and 16 were lost with the last contact at sometime during 1973-1978. Accretion of study data was continued for all patient visits or admissions to July 1980 .

From the follow-up procedures it was determined that 142 of the 390 patients had died before the end of the study, leaving 208 patients that were alive or lost to follow-up. The survival times for all patients were determined as follows: for those 142 patients who died, the survival time was the elapsed time in years between the "entry date" and the date of death. The remaining 248 patients, for purposes of this analysis, were considered lost to follow-up on the date of the last recorded visit to University Hospital. All of the analyses were performed without regard to specific cause of death, treating all deaths as scleroderma deaths.

Because this is a retrospective analysis, several definitions of date of entry were used to insure that any potential biases associated with a particular definition of survival were evaluated. Alternative definitions of "date of entry" include date of first diagnosis, date of first symptom, date of first University of Michigan Hospital visit, and date of initial complete medical evaluation at University Hospital.

*The computer software package BMDP [9] was used to generate the life tables and to perform the Cox analyses and all the associated statistical tests.
For the purpose of describing the survival data for this patient population, the date of initial diagnosis of scleroderma was taken as the date of entry. This designation also permits comparison of findings to those of a number of other studies which performed survival analyses based on date of scleroderma diagnosis [2-5]. Date of diagnosis was not necessarily the date of the first University of Michigan Hospital visit, rather it was the first physician diagnosis of scleroderma. The earliest date was June 1946, the mean date was 1968 , and average age at diagnosis was 42.4 years.

The survival times were analyzed using several methodologies. First, the survival data were analyzed using the life-table method. Survival curves were plotted using the Kaplan-Meier method [6]. Differences between the estimated survival curves for the population subgroups were evaluated using the Mantel-Cox test [7]. $p$-Values are reported for two tailed significance levels.

Within the context of the life-table analyses it was also informative to compare the observed mortality of our patient population to the expected mortality for the same patients using age (at diagnosis), race, and sex specific U.S. death rates.

Finally, the Cox proportional-hazards model [8] was used to analyze the data. ${ }^{*}$ This was done because differences in survival between two subgroups cannot necessarily be attributed to the particular characteristic distinguishing the two groups when other factors that affect survival differ between the groups being tested. The life-table method and the associated statistics for testing equality among survival curves assume that the subgroups being tested are homogeneous with respect to all other factors affecting survival. The Cox proportionalhazards model permits evaluation of the separate effects of multiple demographic and prognostic factors and simultaneously accounts for heterogeneity of subgroups. Moreover, the Cox model provides a stronger test for evaluating the effects of treatment on survival because it allows the statistical comparison of treated and non-treated groups while correcting for differences in demographic characteristics and important associated organ system involvements that are present at the outset of treatment.

With these points in mind, seven indicators of health status or organ system involvement at date of entry were used in addition to age, sex, 
race, and KPAB treatment category as covariates in Cox analyses. Among the clinical elements chosen for analysis with regard to their prognostic potential were: (1) the extent of skin involvement [categorized as to (a) minimalmild, (b) moderate, or (c) marked or severe involvement] (1); (2) hypertension (documented history, systolic $>150 \mathrm{mmHg}$, or diastolic $>$ $90 \mathrm{mmHg}$ ); (3) renal involvement (BUN > $25.0 \mathrm{mg} \%$ or serum creatinine $>1.5 \mathrm{mg} \%$ ); (4) pulmonary fibrosis by chest roentgenogram; (5) cardiac involvement (abnormal electrocardiagram, pericardial effusion, or congestive failure by chest roentgenogram); (6) presence or history of characteristic Raynaud's phenomenon; and (7) esophageal involvement (dysphagia and/or findings associated with scleroderma on barium swallow or manometrics). Correlations among the covariates were evaluated using the Chi-square test for independence in a contingency table.

Statistical differences between models contạining different covariates and significance of covariates within a model were evaluated using a likelihood ratio test distributed Chi square. In addition, the relative powers of the prognostic indicators were evaluated using a stepwise Cox methodology.

\section{RESULTS}

The survival data for this patient population by race, sex, and treatment group are summarized in Table 1. The average age at first diagnosis was 42.4 years while the average age at death was 54.5 years. In addition to being diagnosed at a younger age, Table 1 shows non-white patients dying younger on average than white.

The ratio of females to male patients is 4.4 ; thus, females accounted for $81 \%$ of the total population. The mean age at entry for males and females was 42.0 and 42.4 , respectively. Non-whites accounted for $12 \%$ of the 390 and were on average 6 years younger than whites at first diagnosis, 37.1 compared to 43.1 for whites. The mode of the age distributions occurred in the 40-49 range for all race and sex subgroups.

The treated patients are divided into those adequately and inadequately treated. Patients who took potassium para-aminobenzoate as prescribed for dose and duration were considered "adequately treated" [1]. The usual daily dose was $12 \mathrm{~g}$ (four doses, $3 \mathrm{~g}$ each at meals and bedtime) or $12.5 \mathrm{~g}$ (five doses, $2.5 \mathrm{~g}$ each at

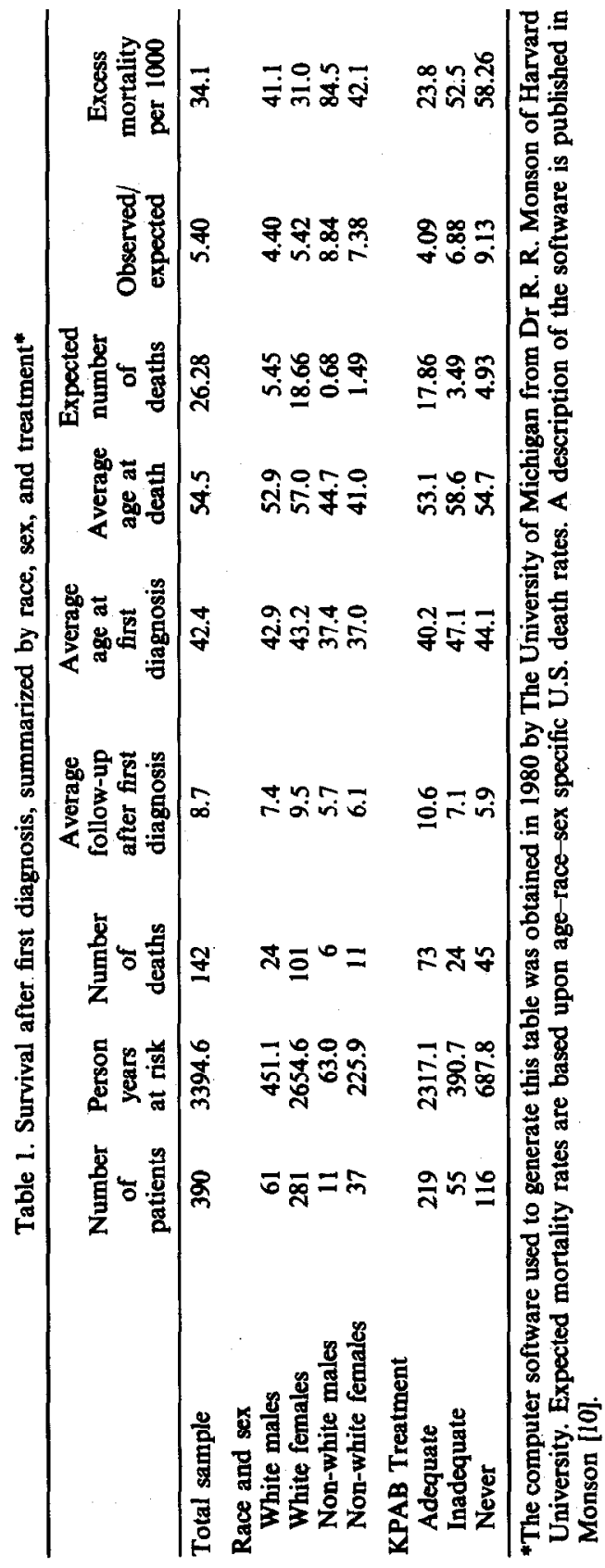


meals, mid-afternoon, and bedtime). In a few instances $10 \mathrm{~g}$ or less per day was prescribed for patients who weighed less than $100 \mathrm{lb}$. Patients who, for whatever reason, took less medication than was prescribed or discontinued treatment on their own after a brief interval were considered to be "inadequately treated" with this medication. Duration of treatment was influenced by extent and severity of skin involvement and/or the presence of pulmonary fibrosis. When treatment with KPAB was initiated, it was begun on average 2.4 years after the diagnosis of scleroderma had been made. At the above prescribed doses the beginning of skin softening may be detected clinically in some patients after about 3 months of treatment, with further softening at 6 months and beyond. Softening is very gradual. Treatment, therefore, has generally been continued as long as there was potential for further skin softening and/or significant pulmonary involvement was present. Thus, adequately treated patients took KPAB for periods ranging from 3 months to as long as 20 years, with the average duration at 4.2 years.

Treated patients accounted for 274 of the study population, and of these 219 were adequately treated. There were 116 patients who were at no time treated with KPAB. Mean age at diagnosis for the never treated group (44.1 years) was 3.9 years older than the adequately treated group ( 40.2 years) and 3 years younger than the inadequately treated group. Of the never treated patients $39 \%$ died during the study period compared to 33 and $44 \%$ for the adequately and inadequately treated groups, respectively. Adequately treated patients died on average 1.6 years younger than the never treated with KPAB patients ( 53.1 vs 54.7 years).

The last two columns of Table 1 compare the observed mortality of these patients to the expected mortality based on standard U.S. age-race-sex specific death rates. The ratio of observed and expected deaths indicates that overall our study population experienced 5.4 times the number of deaths expected the same standard U.S. population. Non-white males show the highest ratio, 8.84 , while white males have the lowest, 4.40 . The excess mortality rate in the last column is defined as [(observed - expected deaths)/person years at risk $\times 1000$, and indicates the rate of mortality relative to the expected rate. While substantially more females tend to develop scleroderma, Table 1 shows that males and particularly non-white males have higher death rates. The data by treatment group show the highest values for the ratio of observed to expected deaths (9.13) and for excess mortality (58.26) occur in the never treated group, while the adequately treated group has the lowest values (4.09 and 23.8).

\section{Life table survival results: demographics}

For the life-table analyses date of entry into the survival study was defined as date of initial diagnosis of scleroderma. A similar analysis was performed using the date of first symptom as reported by the patient. As would be expected, the survival times are longer when using the date of first symptom as the date of entry. However, only the results using date of first diagnosis as date of entry are reported here as the basic conclusions from the analyses were not altered. Table 2 summarizes the life-table analysis by age, sex, and race.

Figure 1 shows the observed survival compared to the expected survival for all $390 \mathrm{pa}-$ tients in our study. Scleroderma patients exhibit decreased survivorship compared to the standard mortality of the U.S. population adjusted for the same demographic characteristics. The observed 5 year cumulative survival rate was $81.4 \%$ and the 10 year rate was $69.4 \%$ (Table 2).

As the scleroderma survival literature has indicated, age at entry is an important determinant of survival. The life-table analysis of survival by age confirms previous findings. Table 2 also compares survival of patients 40 years of age and older at first diagnosis to that of patients under 40 . The older patients have significantly decreased survivorship (Table 2, $p<0.001)$ compared to their younger counterparts. This was to be expected since older patients naturally have higher mortality rates than younger ones.

One method to adjust effectively for the "natural" effects of age on survival is to compare excess mortality rates because expected mortality incorporates natural aging effects. If the disease did not have a differential impact by age then the excess mortality rate would not vary across age groups. However, the excess mortality rate for patients diagnosed after age $\mathbf{4 0}$ is 43.56 while the rate for those diagnosed before age 40 is 18.02. The disease impact on the older group, adjusting for natural aging effects, is thus much more severe. Together the analyses indicate that age contributes to a generally poor 
Table 2. Survival from first diagnosis*

\begin{tabular}{|c|c|c|c|c|c|}
\hline & & Five- & Seven- & & $\begin{array}{c}\text { Test for } \\
\text { differences } \\
\text { between groups }\end{array}$ \\
\hline & $\begin{array}{l}\text { Number } \\
\text { at entry }\end{array}$ & $\begin{array}{l}\text { survival } \\
\text { rate }\end{array}$ & $\begin{array}{l}\text { survival } \\
\text { rate }\end{array}$ & $\begin{array}{l}\text { survival } \\
\text { rate }\end{array}$ & $\begin{array}{c}\text { Mantel-Cox test } \\
(p \text {-value })\end{array}$ \\
\hline Overall & 390 & $\begin{array}{l}81.4 \\
(2.1)\end{array}$ & $\begin{array}{l}77.1 \\
(2.3)\end{array}$ & $\begin{array}{l}69.4 \\
(2.7)\end{array}$ & - \\
\hline Female & 318 & $\begin{array}{l}84.2 \\
(2.2)\end{array}$ & $\begin{array}{l}79.8 \\
(2.5)\end{array}$ & $\begin{array}{l}72.7 \\
(2.9)\end{array}$ & \multirow{2}{*}{0.057} \\
\hline Male & 72 & $\begin{array}{l}69.2 \\
(5.8)\end{array}$ & $\begin{array}{l}65.0 \\
(6.1)\end{array}$ & $\begin{array}{l}54.6 \\
(7.1)\end{array}$ & \\
\hline White & 342 & $\begin{array}{l}82.4 \\
(2.2)\end{array}$ & $\begin{array}{l}78.0 \\
(2.4)\end{array}$ & $\begin{array}{l}70.7 \\
(2.8)\end{array}$ & \multirow{2}{*}{0.154} \\
\hline Non-white & 48 & $\begin{array}{l}74.4 \\
(6.7)\end{array}$ & $\begin{array}{l}70.9 \\
(7.2)\end{array}$ & $\begin{array}{l}59.0 \\
(9.7)\end{array}$ & \\
\hline Age at first diagnosis $>40$ & 240 & $\begin{array}{l}77.2 \\
(2.9)\end{array}$ & $\begin{array}{l}73.0 \\
(3.1)\end{array}$ & $\begin{array}{l}64.0 \\
(3.6)\end{array}$ & \multirow[b]{2}{*}{$<0.001$} \\
\hline Age at first diagnosis $<40$ & 150 & $\begin{array}{c}87.8 \\
(2.8)\end{array}$ & $\begin{array}{c}83.2 \\
(3.3)\end{array}$ & $\begin{array}{l}77.8 \\
(3.9)\end{array}$ & \\
\hline
\end{tabular}

*Standard errors in parentheses.

prognosis in addition to its natural mortality effects.

Gender has also been shown to be an important determinant of survival (Table 2). Males show an unfavorable prognosis relative to females but the difference failed to attain statistical significance $(p=0.057)$ at the $5 \%$ level. There was no significant difference between the mean age at entry for these two groups.

Although non-white patients had decreased survivorship compared to whites the difference was also not significant $(p=0.15)$ (Table 2).

Life table survival results: effect of potassium para-aminobenzoate therapy

These analyses provide strong evidence of the efficacy of KPAB therapy on the survival of

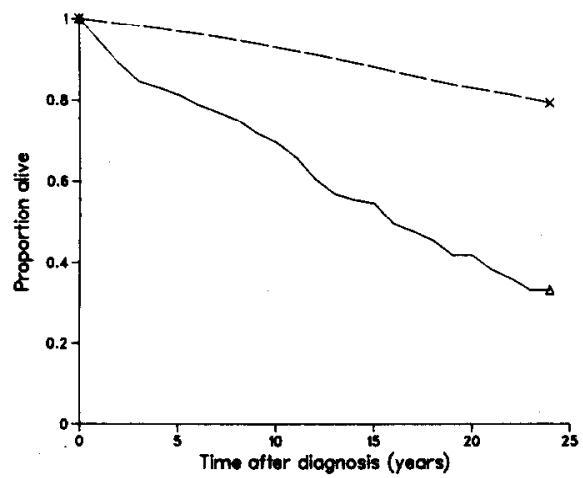

Fig. 1. Survival curves, observed vs expected for 390 scleroderma patients. $\Delta$, Observed; $x$, Expected. patients with progressive systemic sclerosis. In this section the survival curves for the various treatment groups are examined, while in the next section the Cox model will provide a more powerful analytical tool which permits a more comprehensive comparison of treatment groups while adjusting for differences between groups for other factors known to affect survival. Table 3 gives the 5,7, and 10-year cumulative survival rates for the 116 patients who were never treated with KPAB, and for the 274 patients who received $\mathrm{KPAB}$ at some time during their scleroderma course. Those treated with KPAB have a significantly $(p<0.01)$ prolonged life span compared to the group who were never treated with KPAB. The treated group shows 5-year and 10-year survival rates of 85.7 and $73.9 \%$, respectively, compared to 69.8 and $56.6 \%$ for the group that had never been treated with KPAB.

If the treated group is divided into those adequately and inadequately treated, and the estimated survival curve of the adequately treated group is then compared to that of patients who had not received KPAB, the difference is even more pronounced. Figure 2 shows the estimated survival curves for the adequately and never treated groups. The adequately treated group has significantly prolonged survivorship $(p<0.01)$ with 5 -year and 10 -year cumulative survivor rates of $\mathbf{8 8 . 5}$ and $76.7 \%$, respectively.

The importance of adequate treatment with 
Table 3. Survival from first diagnosis* as related to KPAB treatment category

\begin{tabular}{lcccc}
\hline & $\begin{array}{c}\text { Number } \\
\text { at entry }\end{array}$ & $\begin{array}{c}\text { Five- } \\
\text { year } \\
\text { survival }\end{array}$ & $\begin{array}{c}\text { Seven- } \\
\text { year } \\
\text { survival }\end{array}$ & $\begin{array}{c}\text { Ten-year } \\
\text { survival }\end{array}$ \\
\hline Treated with KPAB & 274 & 85.7 & 81.5 & 73.9 \\
& & $(2.2)$ & $(2.5)$ & $(3.0)$ \\
Adequately treated & 219 & 88.5 & 84.5 & 76.7 \\
& & $(2.2)$ & $(2.6)$ & $(3.2)$ \\
Inadequately treated & 55 & 74.0 & 68.6 & 61.9 \\
& & $(6.3)$ & $(6.9)$ & $(7.7)$ \\
Never treated & \multirow{2}{*}{116} & 69.8 & 64.6 & 56.6 \\
& & $(4.8)$ & $(5.3)$ & $(6.0)$ \\
\hline
\end{tabular}

* Standard errors in parentheses.

KPAB is evidenced by comparison of the inadequately treated group to both the adequately and never treated groups. Testing the difference in these estimated survival curves shows a significant difference $(p<0.01)$ between the adequately and inadequately treated groups and no difference $(p=0.91)$ between the inadequately and never treated with KPAB groups.

These treatment groups differ with respect to other factors affecting survival. For example, the mean age of entry for the never treated group was 2.2 years older (44.1 vs 41.9 ) than the group who received KPAB treatment at some time, but this difference in mean ages was not significant $(p=0.18)$. For the adequately treated group the mean age of entry was 40.2 and the 3.9 years age difference between the groups was significant $(p=0.04)$. The Cox analysis, discussed below, is applied to correct for differences in age as well as for other patient characteristics including sex, race, and health status. Before addressing the results of the Cox model analyses it is relevant to note that the effects of age can be controlled by examining the observed to expected mortality since the ex-

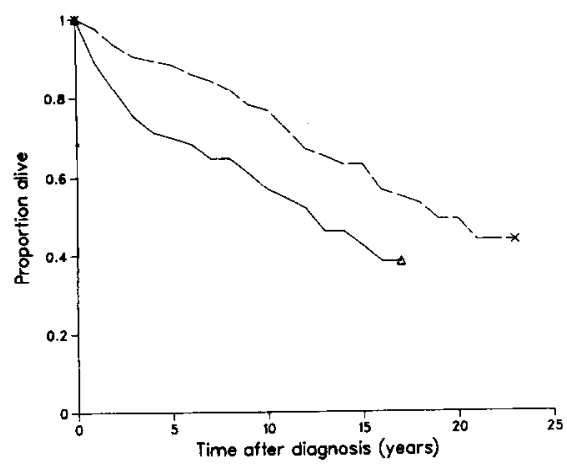

Fig. 2. Survival curves for 335 scleroderma patients by KPAB treatment group: 219 treated adequately with KPAB vs 116 patients who never received KPAB. $\triangle$, Never TX; $x$, Adequate TX. pected mortality rates embody the natural mortality effects of aging. The ratio of observed to expected deaths for the adequately, inadequately, and never treated groups is $4.09,6.88$, and 9.13, respectively (Table 1). The excess mortality rates for the treatment groups have the same increasing pattern $(23.8,52.5$, and 58.26) with the never treated group having the highest rate and the adequately treated patients having the lowest.

This pattern of relative mortality and the Mantel-Cox tests for differences in survival among the treatment groups provide evidence of the positive effect of Potaba ${ }^{\circledR}$ therapy on survival and the importance of adequate dose and duration of treatment.

\section{Cox proportional hazards model results}

Although the life-table analyses have indicated the importance of age, race, sex, and KPAB treatment to the survival of scleroderma patients, these analyses were confounded by the heterogeneity of subgroups with respect to other factors affecting survival such as age or organ system involvement at the onset of treatment. The use of the Cox proportional hazards model permits the comparison of subgroups, and in particular treatment groups, adjusting for other factors known to affect survival.

However, it should be recognized at the outset that caution must be exercised in interpreting the results of the Cox analyses. There are a number of problems inherent in the application of the Cox model to a retrospective study such as this. Ideally, the Cox model will estimate the separate effect of treatment while controlling for patient demographics and health status at the date of entry. On the date of entry in a prospective clinical trial, patients can be evaluated for all relevant health characteristics, randomly assigned to treatment groups, and therapy ini- 
tiated. The date of entry (the time the survival clock begins), the assessement of initial patient health status, and the beginning of treatment are synchronous. In a retrospective study the data do not conform to the ideal clinical trial. In the present study patients were not randomly assigned to treatment groups nor was there a systematic application of clinical tests. There were some patients for whom the clinical information necessary to assess their health status was simply not available. Moreover, patients did not necessarily begin treatment at any systematic time in the course of their disease. However, first University Hospital visit did constitute the date on which a majority of patients received an extensive clinical evaluation and began treatment.

First, results of the Cox model survival analysis controlling only for demographic factors and treatment are given in Table 4. Like the lifetable analyses, the Cox model results reported in Table 4 include all 390 patients. For this analysis, which includes only demographic covariates, date of entry is also the date of diagnosis. Patients are allocated into the treatment groups (either adequate or inadequate) if they received KPAB at some time in the course of their disease. The analysis retains a covariate identifying the inadequately treated group to insure that no bias can result from including these patients as never treated and to demonstrate whether their survivorship differs from the never treated group. All covariates in the analysis except age are dichotomous with values zero and one where the value one for the variable indicates the presence of the covariate (e.g. race is white, sex is male, or received KPAB treatment). Age is measured in years of age at diagnosis and enters linearly into the model. There was no evidence that a quadratic term in age contributed substantially to the model.

The results in Table 4 are consistent with the life-table analyses. Age remains an important determinant of survival with risk increasing $4 \%$ for each year of age at entry $(p<0.001)$. This analysis indicates the importance of gender in survival. Whereas the poor prognosis for males did not attain statistical significance at the $5 \%$ level in the life-table analysis, this model shows an increased risk of $60 \%$ for males over females and the difference attains significance $(p=$ 0.03 ). Prolonged life (a negative coefficient implying a lower hazard rate) is associated with whites relative to blacks and adequately and inadequately treated patients relative to the never treated group. In this specification of the model whites show lower risk but again the difference does not attain significance at the 5\% level. With respect to treatment these results confirm the conclusions reached in the life-table analysis on the importance of dose and duration of KPAB treatment. The adequately treated group shows a hazard rate $51 \%$ lower than the never treated group with the difference being highly significant $(p=0.0004)$. The inadequately treated group, on the other hand, shows a lower hazard rate relative to the never treated with KPAB group and, as in the life-table analysis, the difference is not significant.

The Cox model was next extended to include, in addition to age, sex, race, and treatment with $\mathrm{KPAB}$, seven indicators of health status or organ system involvement at date of entry. As noted above, patients had not been clinically evaluated nor had treatment been initiated at any systematic time in the course of their disease. However, first University Hospital visit did constitute the date on which the majority of patients were extensively evaluated. Moreover, KPAB treatment was initiated within 3 months of that visit for 121 of 219 adequately treated patients. Thus, first University Hospital visit was taken as the date on which potential followup began. Because the Cox analysis corrects for differences in health status at date of entry, the actual date of entry could not precede either the initial clinical assessment or, for the adequately treated patients, the date of initial treatment. Should the date of entry into the Cox analysis have preceded the date of first treatment, then survival time prior to the beginning of therapy would be inappropriately atttributed to a poten-

Table 4. Survival analysis using Cox proportional-hazard model

\begin{tabular}{lcccc}
\hline Variable & \multicolumn{3}{c}{ Standard } & \\
& Coefficient & error & $p$-value & Exp. (coefficient) \\
\hline Age & 0.04 & 0.006 & $<0.0001$ & 1.04 \\
Male & 0.47 & 0.21 & 0.03 & 1.60 \\
White & -0.51 & 0.27 & 0.08 & 0.60 \\
Inadequate KPAB treatment & -0.16 & 0.26 & 0.53 & 0.85 \\
Adequate KPAB treatment & -0.72 & 0.20 & 0.0004 & 0.49 \\
\hline
\end{tabular}


tial treatment effect. Survival for the extended model was, therefore, defined as follows: for all treatment groups survival time prior to first University Hospital visit was excluded from the analysis. For the groups inadequately and never treated with KPAB, date of entry was the first occurrence of a complete clinical assessment at University Hospital. The adequately treated group was similarly defined; however, in addition, the survival clock did not begin until adequate KPAB treatment had been initiated.

It is important to note that in some instances KPAB was often prescribed at one time but for various reasons adequate treatment did not begin for months or in a few cases, years later. For the adequately treated patients the date of first treatment was defined as the date that adequate treatment began regardless of when KPAB was prescribed in the past or how long the patient was inadequately treated before becoming adequately treated. All survival time subsequent to adequate treatment with KPAB was considered survival time under treatment even if treatment was discontinued at some future time.

Not all 390 patients could be included in the Cox analysis. First, patients were excluded who lacked a complete set of clinical data on a single visit or admission to University Hospital. Second, patients with short survival times constituted a potential source of bias since they were effectively precluded from receiving adequate treatment. Thus, patients were excluded if death or loss to follow-up occurred within 3 months of their first University Hospital visit. The following criteria define the sample population for the Cox analysis which included the clinical covariates: (1) patients were included in the sample who had completed tests for the designated indicators (Table 5) within a 2-month span of time of a single visit to University Hospital; and (2) patients were included who were followed at least 3 months after admission to University Hospital.

There were 294 of the 390 patients who had adequate clinical information and duration of follow-up to be included in the sample. Seventysix patients were excluded because they never had complete clinical data on a single visit. An additional 20 were excluded because they either died or were lost to follow-up within 3 months of the first University Hospital visit. First University Hospital visit was the date of entry for 215 of the 294 patients, leaving 79 for whom the necessary data were obtained at subsequent

Table 5. Clinical characteristics at date of entry and treatment distribution of 294 patient with complete clinical data

\begin{tabular}{|c|c|c|c|c|c|}
\hline \multirow[b]{2}{*}{ KPAB treatment } & \multicolumn{2}{|c|}{ Total sample } & \multicolumn{3}{|c|}{$\begin{array}{l}\text { Percent of patients in KPAB } \\
\text { treatment group with indicated } \\
\text { variable }\end{array}$} \\
\hline & Number & Percent & Adequate & Inadequate & Never \\
\hline Adequate & 179 & 60.9 & - & - & - \\
\hline Inadequate & 41 & 13.9 & - & - & - \\
\hline Never & 74 & 25.2 & - & - & - \\
\hline \multicolumn{6}{|l|}{ Demographics } \\
\hline Mean age \pm & $\begin{array}{c}45.5 \\
(14.5)\end{array}$ & - & $\begin{array}{c}44.9 \\
(14.2)\end{array}$ & $\begin{array}{c}47.4 \\
(14.5)\end{array}$ & $\begin{array}{c}46.1 \\
(15.1)\end{array}$ \\
\hline Male & 60 & 20.4 & 20.1 & 14.6 & 24.3 \\
\hline Female & 257 & 87.4 & 88.3 & 92.7 & 82.4 \\
\hline \multicolumn{6}{|l|}{ Clinical involvement } \\
\hline Raynaud's* & 232 & 85.9 & 84.1 & 90.2 & 87.5 \\
\hline Pulmonary & 69 & 23.5 & 22.3 & 29.3 & 23.0 \\
\hline Esophageal & 235 & 79.9 & 80.4 & 80.5 & 78.4 \\
\hline Hypertension $\dagger$ & 72 & 24.5 & 17.3 & 26.8 & 40.5 \\
\hline Renal & 14 & 4.8 & 1.7 & 4.9 & 12.2 \\
\hline Heart & 34 & 11.6 & 10.6 & 7.3 & 16.2 \\
\hline \multicolumn{6}{|l|}{ Skint } \\
\hline Minimum, mild & 60 & 20.4 & 15.7 & 24.4 & 29.7 \\
\hline Moderate & 82 & 27.9 & 25.7 & 29.3 & 32.4 \\
\hline Marked, severe & 152 & 51.7 & 58.7 & 46.3 & 37.9 \\
\hline
\end{tabular}

*The sample size for Raynaud's phenomenon was 270 (24 of the 294 were missing values). Raynaud's phenomenon was excluded from the Cox analysis for reasons given in the text.

$\uparrow$ Indicates the difference among treatment groups is significant at the 0.05 level. \pm Standard deviation in parentheses. 
visits. There were 104 deaths, leaving 239 cases either lost to follow-up or alive at the end of the study.

It is important to note that there were 79 cases who were treated prior to the date of entry either because they were already under treatment when they came to the University Hospital [38] or they were begun on treatment but did not have all the clinical data necessary for inclusion in the Cox extended model until a subsequent visit [41]. These 79 patients were treated for an average of 2.5 years before the date of entry into the Cox analysis. This time of concident treatment and survival which preceded date of entry was excluded in order to meet the criteria of the Cox model.

With the exception of skin involvement and age, all covariates in the model are dichotomous variables with the values of zero and one, where one indicates that the patient has the associated clinical involvement, is white or female, or is adequately or inadequately treated. Age defined as age in years at date of entry, enters linearly into the analysis. The covariate for skin involvement enters into the analysis linearly taking on the values one, two, and three. The value one indicates minimum or mild skin involvement, two indicates moderate, and the value three indicates marked or severe involvement [1]. A graphical evaluation of the proportionality assumption of the Cox model indicated this was an appropriate way to model skin involvement. A similar evaluation for the treatment groups revealed no evidence of nonproportionality.

Table 5 summarizes the clinical characteristics by treatment group of the sample popu- lation used in the Cox analysis. The findings given in Table 5 indicate that patients were not randomly assigned to treatment groups. $\mathrm{Pa}$ tients with higher degress of skin involvement were more likely to receive $\operatorname{KPAB}(p<0.01)$; while those with renal involvement or hypertension were less likely to receive this medication ( $p<0.01$ for both). There are a number of additional statistical correlations among the covariates worth noting; (1) Raynaud's phenomenon is positively correlated with esophageal involvement $(p<0.01)$. (2) Pulmonary involvement is positively correlated with heart involvement $(p<0.01)$ and hypertension $(p<0.01)$. (3) Heart involvement is, in addition, positively correlated with hypertension $(p<0.01)$, and renal involvement $(p<0.01)$. Despite the non-random assignment of cases, the Cox model will adjust for the differences among treatment groups at the date of entry.

Table 6 shows the results of the Cox proportional-hazards model adjusting for demographics, clinical characteristics, and treatment. The analysis reported here excludes the covariate for Raynaud's phenomenon because preliminary analysis consistently showed Raynaud's to be an insignificant prognostic indicator. By eliminating Raynaud's the model gains a degree of freedom and additional cases could be added to the sample because the criteria for inclusion into the sample required complete data for six clinical covariates instead of seven.

As in the previous analysis, Table 6 shows prolonged life is associated with whites relative to non-whites, and inadequate and adequate KPAB treatment relative to the never treated

Table 6. Cox proportional-hazard model results

\begin{tabular}{|c|c|c|c|c|c|}
\hline Covariate & & Coefficient & $\begin{array}{l}\text { Standard } \\
\text { error }\end{array}$ & $p$-value & Exp. (coefficient) \\
\hline \multicolumn{6}{|l|}{ Demographics } \\
\hline Age & & 0.014 & 0.009 & 0.10 & 1.01 \\
\hline Male & & 0.416 & 0.25 & 0.11 & 1.52 \\
\hline White & & -0.203 & 0.33 & 0.55 & 0.82 \\
\hline \multicolumn{6}{|l|}{ Clinical involvement } \\
\hline Esophageal & & 0.449 & 0.29 & 0.10 & 1.57 \\
\hline Pulmonary & & 0.466 & 0.24 & 0.06 & 1.59 \\
\hline Heart & & 0.693 & 0.26 & 0.01 & 2.00 \\
\hline Hypertension & & 0.470 & 0.25 & 0.06 & 1.60 \\
\hline Renal & & 0.476 & 0.38 & 0.23 & 1.61 \\
\hline Skin (by extent) & & 0.429 & 0.15 & 0.002 & 1.54 \\
\hline \multicolumn{6}{|l|}{ Treatment-KPAB } \\
\hline \multirow{3}{*}{$\begin{array}{l}\text { Inadequate } \\
\text { Adequate }\end{array}$} & & -0.208 & 0.35 & 0.55 & 0.81 \\
\hline & & -0.478 & 0.27 & 0.08 & 0.62 \\
\hline & $\frac{\text { Total }}{294}$ & $\frac{\text { Dead }}{104}$ & & $\frac{\text { Censored }}{190}$ & \\
\hline
\end{tabular}


group. The influence of race and of inadequate treatment on survival was in the same direction as were the results in the life-table analysis but did not reach statistical significance. This model indicates that adequate treatment results in a $38 \%$ reduction in the hazard rate but the coefficient is only marginally significant $(p=0.08)$.

A relatively poor prognosis is associated with all of the clinical factors. Table 6 shows the worst prognosis is for those patients with heart involvement with a hazard rate $100 \%$ higher than for those with normal heart function $(p=0.01)$. The extent of skin involvement is also a powerful prognostic indicator of survival having the greatest explanatory power in the model $(p=0.002)$. The more extensive the skin involvement at date of entry, the more reduced is survivorship. The results indicate that patients with marked involvement have hazard rates $54 \%$ higher than those with moderate involvement. Similarly, because the model restricts the difference in hazard rates between the groups to be proportionally constant, the hazard rate for those with moderate skin involvement is $54 \%$ higher than for the group with minimum or mild involvement.

Pulmonary fibrosis increases risk by $59 \%$ $(p=0.06)$. Hypertension leads to a $60 \%$ increased hazard rate over those without hypertension $(p=0.06)$. Renal involvement shows an increased risk of $61 \%$ but was not significant. However, it may be noted from Table 5 that there were only 14 cases who met the criteria for renal involvement in this sample.

The Cox analysis was repeated using the stepwise regression method. In contrast to the results in Table 6 which weighed the relative contribution of each covariate, the stepwise method indicates the group of variables which has the greatest prognostic power, in the order of that power, to explain the variation in survival times. This method showed that the pres- ence of hypertension in scleroderma patients had the strongest prognostic power $(p<0.001)$. Hypertension was followed by cardiac involvement $(p=0.001)$, pulmonary involvement $(p=0.009)$, extent of skin involvement $(p=0.014)$, and renal involvement $(p=0.06)$.

In addition, the Cox analysis reveals that patients with renal involvement or hypertension are relatively high risk patients. Because these patients were also less likely to receive KPAB (especially those with renal involvement), the patient population was separated into two subgroups, those with renal involvement and/or hypertension, and those without. A Cox analysis was then performed on each group separately. The results showed a differential effect of KPAB on the two groups. In the low risk group, i.e. those without renal involvement or hypertension, adequate treatment resulted in a lower hazard rate $(55 \%$ lower than for the never treated group) and a higher level of significance $(p=0.048)$ compared to the total population sample. There was no treatment effect in the high risk group. The results suggest that treatment prevents or delays progression of some aspects of the disease and thereby prolongs survival. However, once renal involvement or hypertension are present the patients face a poor prognosis, notwithstanding treatment with KPAB.

Finally, with regard to the present study, there was no selection as to which patients charts were extracted: availability of records was the determining factor. That this process itself may have introduced bias regarding treatment results was considered. Table 7 summarizes the incident and prevalent cases by KPAB treatment groups. Incident cases were defined as those patients whose diagnosis of scleroderma was made less than 1 year from onset of symptoms. Table 7 shows that the percentage of incident and of prevalent cases are approximately equal across treatment groups.

Table 7. Incident* and prevalent cases by treatment groups

\begin{tabular}{lcccc}
\hline & \multicolumn{3}{c}{ No. by KPAB treatment status } \\
\cline { 2 - 5 } & & Adequate & Inadequate & Never \\
\hline No. patients-total & $390 \dagger$ & 219 & 55 & 116 \\
Incident cases & 137 & 78 & 17 & 42 \\
& & $(36.4 \%)$ & $(31.5 \%)$ & $(37.2 \%)$ \\
Prevalent cases & 244 & 136 & 37 & 71 \\
& & $(63.6 \%)$ & $(68.5 \%)$ & $(62.8 \%)$ \\
\hline
\end{tabular}

*Incident cases diagnosed scleroderma within 1 year after first symptom/sign.

$\uparrow$ Nine cases are missing date for first symptom/sign. 
The mean duration of symptoms to start of treatment for patients adequately treated with KPAB was 5.7 years (range 0-35 yr). For those inadequately treated or never treated the mean duration of symptoms to first University Hospital visit was 8.3 years (range 0-30 yr) and 4.8 years (range $0-45 \mathrm{yr}$ ), respectively. The differences in the means were not statistically significant. Both of these results indicate that there was no bias introduced on the basis of differential assignment of patients with long duration from onset of symptoms.

\section{DISCUSSION}

In the present study, several methods of analysis were applied to assess the survival times for 390 scleroderma patients who were seen at The University of Michigan Hospital over the period 1948 to July 1980 . Although some biases in the analyses still remain there is strong evidence of a KPAB effect on survival. Each of the analyses has indicated prolonged survival for scleroderma patients adequately treated with potassium para-aminobenzoate relative to the inadequately and never treated groups. The analyses have also shown prolonged survival for the entire population relative to other studies of scleroderma patients. Cumulative survival rates for the entire group were $81.4 \%$ at 5 years, $77.1 \%$ at 7 years, and $69.4 \%$ at 10 years. Medsger et al. [3] performed life-table analysis of survivorship for 309 scleroderma patients and found the cumulative survival to be $35 \%$ at seven years. In a separate study of 358 scleroderma male patients in veterans hospitals, $\mathrm{Me}$ dsger and Masi [4] found the cumulative survival rate to be 35 percent at seven years. The largest group of patients for whom survival rates are available is that of Tuffanelli and Winkelman [10] who found 5-year survival for $70.3 \%$ and 10 -year survival for $58.9 \%$ of patients traced.

Several factors may have contributed to the prolonged survival of our patients. First, the survival differences may be due in part to differences in definition of entry into the study. Like Bennett et al. [2], our life-table analyses define entry into study as the date of initial scleroderma diagnosis.

Second, the fact that our patients were diagnosed younger on average than other large reported series, with the exception of the study by Tuffanelli and Winkelman may have also contributed to the difference in survivorship. The mean age at entry for Bennett et al. was
46.2 years, 40.3 for Tuffanelli and Winkelman [5], while for the present study the mean age of entry was 42.5 years.

Finally, KPAB therapy has also contributed to higher survival rates in the present study. If we compare the cumulative survival rates of the never treated group with those in the literature the similarity is much greater. This is particularly true when we compare the never treated with KPAB group to the study of Bennett et al. [2]. The 5-year and 10-year survival rates for the group never treated with KPAB are 69.8 and $56.6 \%$, respectively, compared to 73 and $50 \%$, reported by Bennett et al. When the group treated with KPAB is separately analyzed (Table 3), 5-year survival is $88.5 \%$ and 10 -year survival is $76.6 \%$ for adequately treated patients. The difference in survival for patients adequately treated with KPAB and those who have at no time received this medication was statistically significant $(p<0.01)$.

The effect of KPAB on prolongation of survival was also evident in comparisons of observed to expected mortality for these patients. For example, the excess mortality rate was $\mathbf{2 3 . 8}$ for those adequately treated with KPAB as contrasted to 58.26 for those who never received it.

The present study confirms the results in previous studies with respect to the association between age and sex and survival. In our lifetable analysis, the subgroup where age is greater than 40 at diagnosis exhibited significantly decreased survivorship compared to those under 40 as was found by Bennett et al. [2]. Similarly, males had a significantly poorer prognosis for survival compared to that of females.

Medsger et al. [3] noted decreased survival for blacks in the early years of their study, but did not find the race difference significant over the entire period of follow-up. Similarly our nonwhite patients showed decreased survival relative to their white counterparts, but the difference was not statistically significant at the $5 \%$ level.

Because of the important implications of the foregoing findings with respect to prolongation of survival consequent to KPAB therapy, it was deemed important to utilize the Cox proportional-hazards model in an effort to assess the relative importance of various covariates in determining survival. First, when the Cox model was applied to the entire population measuring survival from the date of diagnosis and only adjusting for demographics and treat- 
ment, the results were statistically significant for age $(p<0.001)$, gender $(p=0.03)$, and adequate treatment with $\mathrm{KPAB}(p=0.002)$ while the effect of race was only marginally significant $(p=0.06)$. The Cox model was next applied to the analysis of six clinical variables in addition to age, race, gender, and treatment (KPAB). It should be kept in mind that the Cox model is suited to prospective studies and that its application to this retrospective analysis required a number of adjustments which may well have biased the results. In particular, the correlations between treatment and the clinical variables for renal, hypertension, and skin involvement indicate that patients were not randomly assigned to treatment groups. Several additional points need to be emphasized. First, it should be noted with respect to the negative correlation for $\mathrm{KPAB}$ and renal involvement, that since the drug is excreted via the kidneys it is generally not administered to patients with significant renal involvement. Second, patients with higher degrees of skin involvement were more likely to receive $\mathrm{KPAB}$. Third, applying a model that adjusts for baseline clinical involvement required the exclusion of survival time prior to the patient's complete clinical evaluation. A significant amount of survival time for patients on adequate treatment was thereby excluded. There were of course a number of other correlations among the clinical covariates. All of these variables and correlations are to be separately examined in detail and will therefore not be discussed here.

Despite the difficulties and the inherent problems of retrospective studies there were some interesting results produced by this analysis. Pulmonary $(p=0.06)$, heart $(p=0.01)$, and extent of skin involvement $(p=0.002)$ prove to be important prognostic indicators of survival. Adequate treatment with KPAB still indicates prolonged survival with marginal significance. Age, gender, and race are not significant factors in determining survival as found by this method. Moreover, application of the stepwise regression method with the Cox analysis altered the order of explanatory power of the prognostic variables.

The Cox analysis finding that extent of skin involvement per se is a significant prognostic variable raises the possibility that extent of skin involvement may reflect a magnitude of metabolic stress or derangement not previously appreciated. The survival data presented herein suggest that treatment with KPAB which was initially directed toward softening of involved skin is indeed associated with improved survival.

The prolongation of life associated with KPAB therapy can be linked to its more direct effect on organ system involvement. The results suggest that treatment prevents or delays the progress of some aspects of the disease. The patient population was separated into two groups, those with renal involvement and/or hypertension and those without. A separate Cox analysis performed on each group revealed a differential treatment effect. The effect of KPAB therapy was significant in the low risk group while treatment was ineffective for those who had already developed renal involvement and/or hypertension.

In a separate paper [1] KPAB has been shown to soften thickened, fibrotic skin changes in patients with scleroderma. The effect of KPAB on skin of scleroderma patients however, may be only one aspect of its improvement on survival. Analyses of pulmonary findings and changes in the course of follow-up of patients in this study group have shown a significant effect of KPAB therapy on vital capacity and a lesser though favorable effect on carbon monoxide diffusing capacity [11].

It is of relevant interest to note a recent publication by Steen et al. [12], which reported on the treatment of scleroderma with D-penicillamine (DPA). These authors compared 73 patients treated with D-penicillamine for a minimum of 6-months with 45 patients who did not receive this drug. Survival by the life-table method was increased for the treated group ( 88 vs $66 \%, p<0.05$ ). The average age for the DPA treated patients was 45.3 years while that of the comparison group was 48 years. Patients who had the disease for more than 3 years were excluded from their study as they were considered to have "irreversible fibrosis of skin and viscera". Since we had made no such exclusions in the present study, our population was re-analyzed and showed that 65 of our 390 cases had been diagnosed as having scleroderma for more than 3 years on the first visit to University Hospital. When these 65 patients were excluded from the life-table analysis there was a small increase in the significance for KPAB treatment.

The Cox model was also used to investigate whether the time interval in years from diagnosis to the first University Hospital admission or visit had any bearing on the patient's survival. 
The Cox proportional-hazards model showed that, adjusting for the covariates in Table 6, the interval before hospital admission did not influence survival (less than $1 \%$ reduction in risk, $p=0.92$ ). Clearly, acceptance into treatment programs should not be limited arbitrarily on the basis of duration of scleroderma.

Finally, it may be noted that the chronic use of large doses of KPAB in scleroderma was based on the empirical observation of an associated skin softening effect. The present study yields evidence that skin as well as internal organ-system involvement have prognostic significance. In addition, these retrospective analyses indicate an improved survival from treatment with KPAB. It is believed that the findings in these and related analyses of this group of 390 patients will help to identify areas upon which to focus future clinical studies of scleroderma.

Acknowledgements - The authors express their indebtedness and grateful appreciation to Miss Barbara A. Stoner who carried out the enormous correspondence and telephonic burden associated with this study. They also thank Miss Patricia A. Gastonguay and Mrs Lynn Warren of the University Hospital Medical Information Department for assistance throughout the medical records phase of this study.

Grant support was in part by numerous contributions given in memory of scleroderma patients, and various other donors including Glenwood Inc., Tenafly, New Jersey; Vector Research Inc., Ann Arbor, Michigan, generously provided logistical support. Direct patient research referred to in this and related reports was aided by the Clinical Research Unit (CRU), The University of Michigan Medical School, supported by Grant 2M 01 RR-42-10. Protocols involving clinical investigations were performed in the CRU under the general title "Long Term Multi-system Mani- festations of Scleroderma", after prior review and approval by the Institutional Review Board of The University of Michigan Medical Center.

\section{REFERENCES}

1. Zarafonetis CJD, Dabich L, Skovronski JJ, DeVol EB Wolfe R, Negri D. Retrospective studies in scleroderma: Skin response to potassium paraaminobenzoate therapy. Submitted.

2. Bennett $\mathbf{R}$, Bluestone $\mathbf{R}$, Holt PJL, Bywaters EGL. Survival in scleroderma. Ann Rheum Dis 1971; 30: 581-588.

3. Medsger TA Jr, Masi AT, Rodman GP, Benedek TG, Robinson H. Survival with systemic sclerosis (scleroderma): a life table analysis of demographic and clinical factors in 300 patients. Ann Intern Med 1971; 75: 369-376.

4. Medsger TA Jr, Masi AT. Survival with sclerodermaII: a life-table analysis of clinical and demographic factors in 358 male U.S. veteran patients. J Chron Dis 1973; 26: 647-660.

5. Tuffanelli DL, Winkelmann RK. Systemic scleroderma: a clinical study of 727 cases. Arch Derm 1961; 64: 359-371

6. Kaplan EL, Meier P. Nonparametric estimation from incomplete observations. J Am Stat Assoc 1958; 53: 457-581.

7. Mantel N. Evaluation of survival data and two new rank order statistics arising from its consideration. Cancer Chemother Rep 1966; 50: 163-170.

8. Cox DR. Regression models and life tables. J Stat Soc B 1972; 34: 187-220.

9. BMDP Statistical Software. Department of Biomathematics, University of California, Los Angeles: University of California Press; 1981.

10. Monson RR. Analysis of relative survival and proportional mortality. Comput Biomed Res 1974; 7: 325-332.

11. Zarafonetis CJD, Dabich L, DeVol EB, Skovronski, JJ, Negri D. Retrospective studies in scleroderma: pulmonary findings and influence of potassium paraaminobenzoate. Submitted.

12. Steen VD, Medsger TA Jr., Rodman GP D-Penicillamine therapy in progressive systemic sclerosis (scleroderma): a retrospect analysis. Ann Intern Med 1982; 97: 652-659. 